\title{
Do Transmission Mechanisms or Social Systems Drive Cultural Dynamics in Socially Structured Populations?
}

\section{Citation}

Nunn, Charles Lindsay, Peter H. Thrall, Kevin Bartz, Tirthankar Dasgupta, and Christophe Boesch. 2009. Do transmission mechanisms or social systems drive cultural dynamics in socially structured populations? Animal Behaviour 77(6): 1515-1524.

\section{Published Version}

doi:10.1016/j.anbehav.2009.02.023

\section{Permanent link}

http://nrs.harvard.edu/urn-3:HUL.InstRepos:4317716

\section{Terms of Use}

This article was downloaded from Harvard University's DASH repository, and is made available under the terms and conditions applicable to Other Posted Material, as set forth at http:// nrs.harvard.edu/urn-3:HUL.InstRepos:dash.current.terms-of-use\#LAA

\section{Share Your Story}

The Harvard community has made this article openly available.

Please share how this access benefits you. Submit a story.

\section{Accessibility}



structured populations?

10 Charles L. Nunn ${ }^{1,2,3^{*}}$, Peter H. Thrall ${ }^{4}$, Kevin Bartz $^{5}$, Tirthankar Dasgupta ${ }^{5}$ and Christophe Boesch ${ }^{2}$

11

12

13

$14{ }^{1}$ Harvard University, Department of Anthropology, Cambridge MA 02138 (tel.: 617-495-4710, fax: 617 -

15 496-8041, email: cnunn@oeb.harvard.edu), ${ }^{2}$ Max Planck Institute for Evolutionary Anthropology, D-

1604103 Leipzig, Germany; ${ }^{2}$ University of California, Department of Integrative Biology, Berkeley, CA

17 94720-3140 USA $;{ }^{4}$ CSIRO Plant Industry, GPO Box 1600, Canberra ACT 2601, Australia; ${ }^{5}$ Harvard

18 University, Department of Statistics, Cambridge MA 02138.

19

$20 *$ Author to whom correspondence should be addressed.

21

22 Word count: 9085 (including all references and figure legends)

23 
23 Abstract: Cultural traits spread via multiple mechanisms among individuals within social groups,

24 including transmission biases which occur when subordinates copy from dominants (prestige

25 transmission) or common cultural trait variants are favored over rare ones (consensus transmission). Most

26 animal populations are subdivided into social groups where cultural learning occurs, yet theoretical

27 studies of cultural trait transmission have tended to focus on within-group transmission dynamics. Thus,

28 we lack an understanding of the factors that influence the spread of cultural traits in socially structured

29 populations. We developed an agent-based model of cultural transmission in which a trait arises in one

30 individual and either persists until a stable population equilibrium is reached or goes extinct. With this

31 model, we systematically varied group size, rates of dispersal among groups, mortality rates, transmission

32 characteristics, the benefit of the cultural trait (including possibly negative benefits, i.e. costly traits), and

33 whether individuals disperse locally or randomly to any group. We used generalized linear models to

34 examine how changes in these parameters influence the probability of trait extinction, equilibrium

35 prevalence, and the time to equilibrium. Four traits increased the probability of extinction: smaller group

36 size, higher background mortality, lower transmission rate, and more costly traits. Local dispersal and

37 biased transmission mechanisms (prestige and consensus) had no significant impacts on extinction

38 probability, and similar patterns were found for equilibrium prevalence. By comparison, we found that a

39 lower dispersal rate and local dispersal slowed the time required for a trait to reach equilibrium, as did

40 smaller groups, lower transmission rates, and lower costs. Although increasing costs increased extinction

41 rates, even costly traits sometimes persisted in the simulated populations. Collectively, these analyses

42 provide new insights into the dynamics of cultural traits in socially structured populations, including that

43 prestige and consensus transmission can have weaker effects than other factors associated with

44 demographic and social conditions. In addition, local dispersal and a lower dispersal probability reduced

45 the rate of trait spread but not its prevalence in the population.

47 Keywords: social systems, culture, transmission, individual-based model, social learning. 
A striking feature of human evolution is the incredible diversity of cultures that exist around the world.

For example, linguists have counted over 6,000 languages (Gordon 2005), and humans are thought to

50 practice more than 4,300 religions (faith groups). Many human cultural traits are likely to be adaptive,

51 such as those related to resource allocation and health practices, and are thus subject to natural selection

52 (Mesoudi et al. 2004). Other cultural traits, such as decorations on pottery, are probably driven less by

53 natural selection, but they may provide social or sexual benefits that indirectly translate to higher

54 reproduction. Some persistent cultural traits in humans are even associated with costs. For example, a

55 celibate priesthood dramatically reduces the reproductive success of individuals that become priests,

56 while scarification, excision and circumcision increase the risks of lethal infections, especially in societies

57 living without access to safe medical practices. Potential cultural traditions also have been documented in

58 many nonhuman systems, including nut cracking in chimpanzees (Boesch et al. 1994; Boesch and

59 Boesch-Achermann 2000), potato washing in Japanese macaques (Kawai 1965), and New Caledonian

60 crows that use tools to obtain invertebrates from the vegetation (Hunt 2003). Understanding the spread of

61 cultural traits in non-human systems could provide insights to human evolution and the factors leading to

62 the explosive growth of cultural traits in the human lineage.

A critical question in studies of cultural evolution involves features that impact the dynamics of

64 cultural traits, both in terms of the proportion of individuals that express the trait and the rate at which the

65 behavior spreads through a population. In addition to the cost or benefit of the trait in question, two

66 factors are thought to be important to the spread of cultural traits: the mechanism by which behaviors are

67 learned and the social context in which transmission takes place. First, concerning mechanisms, cultural

68 transmission usually occurs through social learning, in which an individual learns a new behavior by

69 watching other individuals perform the behavior. In nonhuman primates, for example, social learning has

70 been proposed in the case of potato washing in Japanese macaques and nut-cracking in chimpanzees

71 (Kawai 1965; Boesch and Boesch-Achermann 2000). Importantly, different models of cultural

72 transmission may operate, depending on the social system in which the individual is embedded and the

73 mechanisms by which traits are acquired. For example, individuals may be more likely to copy the

$$
\text { Nunn et al., p. } 3
$$


74 behaviors of more dominant individuals, which would be adaptive if dominants possess behavioral traits 75 that made them more successful (Boesch and Tomasello 1998; Boyd and Richerson 1985; Henrich and 76 McElreath 2003). Similarly, individuals may be more likely to adopt traits when more individuals in the 77 group express the trait through a "conformity" or "majority rule" mechanism (Boesch and Tomasello 78 1998; Henrich and McElreath 2003, hereafter called consensus transmission). While these transmission 79 biases are not mutually exclusive, neither are they completely congruent. Second, social context is likely to be important for the spread of cultural traits. At the population

81 level, most primates and humans live in socially structured populations, and the limited evidence

82 available suggests that cultural traits tend to spread more commonly among members of the same social 83 group than between groups (Kawamura 1959; Boesch 2003; Leca et al. 2007). Within social groups, the 84 rate of cultural transmission is expected to be higher when group sizes are larger, with larger numbers of 85 more tolerant individuals providing more opportunities for invention and social learning (van Schaik et al. 86 1999). Opportunities for learning can be modified by other factors, such as proximity of individuals and 87 their capacity for social learning (van Schaik and Pradhan 2003). Mortality rates and movement between 88 social groups can also be important in a socially structured population. If dispersal occurs only between 89 neighboring groups and at a low rate, for example, then the trait in question may take longer to establish 90 in the larger population, and will thus be more prone to cultural extinction if the group is lost due to other

91 factors. Similarly, if individuals who possess a costly cultural trait die at a higher rate, fewer other

92 individuals will have an opportunity to learn the skills that are needed to express the behavior.

93 In this manuscript, we use an agent-based model (Grimm and Railsback 2005) to investigate how 94 cultural traits spread through animal social systems, focusing in particular on features involving group 95 size, dispersal, and background mortality (i.e., a death rate that is independent of expressing the cultural 96 trait). We also examine how different mechanisms of social learning - specifically involving prestige and 97 consensus models - influence the spread of cultural traits, and how different probabilities of acquiring the 98 trait and the selective benefits (or costs) of the trait affect transmission dynamics. The model is spatially 
99 explicit and incorporates three social transmission mechanisms, and individuals can disperse either locally

100 (a spatial model) or randomly to any of the groups (a non-spatial model). In addition, the model allows

101 for variation in group size, dispersal rates, mortality, and the selective benefit (or cost) of the cultural trait

102 (expressed by adjusting the baseline mortality rate among individuals with the trait). The model can

103 therefore be applied to study cultural traits in a wide range of systems in which individuals live in socially

104 structured populations, including humans, non-human primates, and other animals. Our work adds to a

105 growing number of agent-based models of cultural trait transmission, including in the context of foraging

106 (van der Post and Hogeweg 2006, 2008) and the spread of traits through social networks (Franz and Nunn 107 2009).

108 Social learning is a key component of the model. We call the two roles in this exchange the

109 observer, who learns the behavior, and the performer, who exhibits the behavior and therefore serves as

110 the role model for social learning to take place. We investigated three different transmission mechanisms

111 (Boesch and Tomasello 1998; Henrich and McElreath 2003). The first transmission mechanism, referred

112 to as the random transmission model, is the simplest. In this scenario the probability of cultural

113 transmission between two individuals is independent of sex, social affiliation, the proportion of group-

114 mates with the trait, and dominance rank. The other mechanisms represent modifications of the random

115 model. In the prestige transmission model, transmission probabilities are positively correlated with the

116 dominance rank of the individual expressing the trait (Boyd and Richerson 1985; Henrich and Gil-White

117 2001). Consensus transmission addresses the importance of social conformity, with increasing probability

118 that an individual adopts a trait as the proportion of group-mates expressing the trait increases (Boyd and

119 Richerson 1985; Henrich and Boyd 1998). We designed the simulation model so that the mean rate of

120 transmission would be approximately equal across the three transmission mechanisms.

121 We investigated four main questions regarding the relative impacts of social system and

122 transmission characteristics on the spread of cultural traits. In particular we were interested in how these 
123 features interact to determine the probability of extinction, the equilibrium proportion of individuals with 124 the trait, and the time to equilibrium:

125 1. Does local dispersal in a spatially explicit model (i.e., local dispersal) produce different outcomes 126 than a non-spatial model, in which dispersing individuals can move to any social group? Random 127 movement from one group to any other group increases the probability that a dispersing individual 128 with the trait will land in a group that has yet to experience the trait. Once within a group, it is 129 expected to spread rapidly. Thus, random movement should increase the rate of trait spread and favor 130 the establishment of traits in the population. In contrast, local dispersal should slow the rate of 131 cultural dispersion at the population level. Less is known about how local dispersal impacts the 132 prevalence of a trait or its probability of extinction, but we expect that spatially localized traits are 133 more likely to go extinct through stochastic processes.

134 2. Do cultural traits spread more rapidly - and reach higher prevalence - in populations composed of 135 larger social groups, or in populations characterized by higher rates of individual movement among 136 groups? These two social parameters could interact, with larger groups potentially producing more 137 migrants that carry the trait to other groups. Here, we focus on actual movement of individuals 138 between groups (migration), thus assuming that casual observation of individuals in neighboring 139 groups is insufficient for social learning to occur (cf. Boyd and Richerson 2002).

140 3. How does mortality impact the prevalence of a cultural trait in a population? In epidemiological 141 models, higher rates of mortality remove individuals carrying a disease from the population, making 142 it more difficult for the pathogen to become established and reducing overall prevalence (Anderson 143 and May 1979; Thrall et al. 2000). Similar principles should apply to cultural traits. Thus, increased 144 background mortality - i.e., mortality that is independent of the expression of the cultural trait 145 should negatively impact the equilibrium prevalence of the trait. The selective advantage of cultural 146 traits should modify these patterns. Higher benefits (holding costs constant) should lead to lower 147 mortality among individuals with the trait and result in more opportunities for the trait to spread. 
Traits with a net cost should lead to the opposite pattern, resulting in lower prevalence and increased

149 probability that the trait will go extinct.

150 4. How do social learning mechanisms influence the spread of cultural traits? One aspect of social

151 learning involves the probability that a trait will spread from one individual to another. A higher rate

152 of transmission $(\beta)$ could increase prevalence, or reduce the time until equilibrium prevalence is

153 reached. Another aspect of social learning involves the transmission mechanisms discussed above,

154 which effectively modify $\beta$ based on individual characteristics (prestige transmission) or prevalence

155 of the trait in a group (consensus transmission). Compared to a random model, do cultural traits

156 spread more rapidly or reach higher equilibrium prevalence under a prestige or consensus model?

\section{METHODS}

160 Simulation Model Structure

161 We developed a simulation model using the computer package MATLAB (version 7, Natwick,

162 Massachusetts) to simulate the spread of an introduced cultural trait in a socially structured population.

163 The basic design of the model was developed as part of a previous investigation of the impact of host

164 social group structure on the spread of an emerging infectious disease (Nunn et al. 2008). In that study, an

165 initial infection was introduced into a population of susceptible hosts. Individuals that died from disease

166 were not replaced (as is typical of wildlife epidemics), and group composition was allowed to depart from

167 initial conditions as animals died or dispersed from groups. Here, we extend the model to study the spread

168 of culturally inherited traits by including three different transmission mechanisms and adaptive value to

169 the trait (i.e., positive or negative net benefits, representing a beneficial or costly trait, respectively).

170 We were particularly interested in examining the spread of cultural traits in a spatial context,

171 given that previous studies on infectious disease have shown that spatial structure can significantly impact

172 disease dynamics and longer-term evolutionary processes (Thrall and Antonovics 1995; Gandon et al. 
173 1996; Boots and Sasaki 1999; Roy and Kirchner 2000; Carlsson-Graner and Thrall 2002; O'Keefe and

174 Antonovics 2002). For each simulation run, groups of individuals were formed based on user-specified

175 values for group size. Groups were distributed on a 12 x 12 matrix (i.e., 144 groups on a square lattice)

176 and formed as random draws from a Poisson distribution assuming an equal number of males and

177 females. Deaths, births and dispersal of individuals will tend to cause the initial social conditions to drift

178 over a simulation run, especially when simulations are run for many time steps. To deal with this issue,

179 we retained a matrix of the initial numbers of males and females in each group. This "initiating matrix"

180 was used to stochastically adjust probabilities associated with demographic parameters (birth and

181 dispersal) to help maintain initial conditions for each group throughout a simulation run.

The cultural trait was initiated in a single individual, and the trait was allowed to spread through

183 the population in discrete time steps. In each time step, an individual remained in its original group or

184 dispersed to other groups in the population, as determined by the probability of dispersal per time step.

185 We assumed that dispersing individuals lacked contact with conspecifics. We further assumed that

186 dominance rank of a migrant equaled the rank of that individual in the previous group and that this rank

187 did not impact the probability of emigrating or immigrating. Individuals that dispersed were not allowed

188 to enter groups from which they had most recently departed. The simulation was allowed to run until the

189 cultural trait either went extinct in the population or the prevalence of the cultural trait stabilized at a non-

190 zero value.

191

192 Mechanisms of Cultural Transmission

193 Cultural traits spread by social learning within groups, and the probability of transmission $(\beta)$ represents 194 the per-contact probability of an observer acquiring a cultural trait from an individual that expresses the 195 behavior. Mechanistically, $\beta$ encapsulates the combined probability that one individual exhibits the trait 196 while another naïve individual can view and potentially learn from the performer, including the time 197 needed for the observer to learn techniques associated with performing the trait. Thus, lower values of $\beta$ 
could represent behaviors that are more complex (and thus more difficult to learn) or behaviors that are

199 performed more rarely. Individuals that acquire the trait serve as performers in the next time step, and

200 agents retain the trait throughout their lives in a simulation run. In our model, the selective benefits (or

201 costs) of cultural traits are expressed by altering the background probability of death (see below).

202 In the random model, contact rates and per-contact probabilities of transmission were equal

203 among all individuals in a social group regardless of dominance rank and the proportion of individuals

204 exhibiting the trait. Thus, contact rate increased with group size, analogous to predictions from standard

205 mass-action epidemiological models (May and Anderson 1979; Anderson and May 1981). Thus, contacts

206 within groups are assumed to have no spatial restrictions, in comparison to contacts between groups

207 (where contact can only occur through dispersal). Analytically, the probability of a susceptible individual

208 not acquiring the trait as a result of contacts with members of its group is equal to $(1-\beta)^{I}$, where $I$

209 represents the number of individuals in a social group expressing the cultural trait. Thus, the overall

210 probability that an individual learns the trait from one or more performers in a time step is given by 1 - (1

$211-\beta)^{I}$.

212 The prestige model calculates the individual probability that a trait spreads between individuals

213 based on the rank of the performer, under the assumption that observers prefer to copy more dominant

214 individuals within the population, including the possibility that animals possess simple heuristics in which

215 subordinates emulate dominants as a way to learn successful foraging, competitive and hunting behaviors

216 (Boyd and Richerson 1985; Boesch and Tomasello 1998; Henrich and McElreath 2003). At the time of

217 group formation, individuals were assigned dominance ranks $\left(d_{i}\right)$ using values from a uniform

218 distribution. Use of a uniform distribution was preferred to the normal or other distributions because it

219 captures the essence of dominance as a linear ranking, while also allowing some fine differences among

220 individuals in rank. In the process of simulating the spread of cultural traits in the prestige model, user-

221 defined values of $\beta$ were adjusted as follows for spread of a trait from performer $i$ to observer $j$ : 


$$
\beta^{\prime}=\beta\left(0.01+1.98 r_{i}^{\prime}\right)
$$

225 where $r_{i}$ is the standardized rank of the performer, with standardization of ranks within each group

226 ranging from 0 to $1\left[r_{i}^{\prime}=\left(r_{i}-r_{\min }\right) /\left(r_{\max }-r_{\min }\right)\right]$. This procedure gives a range of values for $\beta^{\prime}$ of 0.01 to

2271.99 times the user-specified $\beta$, with the midpoint centered on the user-specified value $\beta$. In this way, the 228 individual with the lowest possible dominance rank $(=0)$ had an adjusted $\beta$ greater than zero $\left(\beta^{\prime}=0.01\right)$,

229 thus preventing deterministic extinction of the trait if the first performer of the trait happened to be the 230 lowest ranking individual in a group. When $\beta>0.5$, the probability of transmission could exceed 1 for

231 higher-ranking individuals. As our values of $\beta$ were always less than 0.04 (Table 1), this should have no

232 effect on model outcomes. Although a stronger version of the prestige model might not allow

233 transmission from the lowest ranking performer to occur, it is worth noting that in our model, the

234 probability of transmission for the lowest ranking individual is two orders of magnitude smaller than a

235 middle-ranking individual; thus, rank has substantial effects on the probability of transmission. In one run

236 of the simulation using the prestige model, we found that the normalized dominance rank of the performer

237 was higher than the observer $(\mathrm{t}=37.7, \mathrm{n}=427$ transmission events, $\mathrm{P}<0.0001)$, with the average rank of the 238 source 0.667 and the average of the recipient 0.495 . For the other transmission models, both performer 239 and observer had similar ranks (average of 0.50).

240 In the consensus model, transmission rates were adjusted based on the percentage of individuals

241 in the group that expressed the trait, $p$. The adjusted $\beta$ was calculated using a linear transformation:

$$
\beta^{\prime}=\beta+(p-0.5) \beta
$$

245 Thus, when less than $1 / 2$ of the group members expressed the trait, $\beta$ was adjusted downwards, and when 246 the majority of individuals expressed the trait, $\beta$ was adjusted upwards. It is important to stress that only 247 the per-contact probability of transmission parameter $(\beta)$ was adjusted, and this reflects the probability of 
transmission between two individuals in the same group; the mass action effect of increasing numbers of

249 'culturally infected' individuals represents an independent effect that tends to increase the spread of traits

250 as more individuals in a group acquire the behavior.

251 The consensus and prestige models were designed to be as simple as possible in their

252 implementation and to have equal average probabilities of transmission, thus allowing comparison among

253 the different transmission models. We acknowledge, however, that different mechanisms of maintaining a

254 constant "average" transmission rate are possible and could produce dynamics that differ from those

255 reported here. We consider this in more depth in the Discussion, along with alternative forms of biased

256 transmission that could be investigated in the future.

258 Maintaining Starting Conditions: Deaths, Births and Dispersal

259 The causes of death were identified during a simulation run as being due to background mortality $(\mathrm{m})$,

260 such as predation and old age, or the presence of a costly cultural trait (as this increased mortality rates

261 through a linear transformation of background mortality). An individual that died from natural causes was

262 replaced by an individual of the same sex. Newly generated healthy individuals were placed in one of the

263 existing groups with a probability that was adjusted according to how current group composition

264 compared to the initiating matrix. If the number of individuals of the sex of the individual being replaced

265 was less than the initiating values for that group, then the probability of assignment was increased. The

266 new group was then determined based on a random draw from a list of all groups, with each group listed

267 once and groups that were deficient given an additional entry. Thus, individuals could be added to any of

268 the groups, but the addition was more likely if the group exhibited a deficit in the number of individuals

269 of that sex, relative to the initiating matrix. As in our previous model (Nunn et al. 2008), we assumed that

270 mortality rates are independent of age and that deaths attributed to a costly trait were not replaced by new

271 individuals (as might be expected if populations are unable to respond demographically to these losses in

272 the time horizons simulated here). 
274 selection on cultural traits acts by increasing or decreasing mortality. The mortality rate of individuals

275 with the cultural trait was multiplied by a selection multiplier, $s_{m}$, which was user-defined and ranged

276 from 0.001 to 2 (Table 1). Thus, selective benefits produced a death rate that was as low as $1 / 1000$ of the

277 baseline mortality $\left(s_{m}=0.001\right)$, and selective costs could increase baseline mortality by as much as 2

278 times $\left(s_{m}=2\right)$. In exploratory simulations, values of $s_{m}>2$ tended to result in rapid and consistent

279 extinction of the trait.

280 We also varied the rate of dispersal, which was measured as the per-day probability that an

281 individual disperses from a group (Table 1). We assumed that dispersal was more likely for groups in

282 which the number of individuals of a particular sex was above the initiating values for the number of

283 individuals for that sex, thus using a procedure similar to that described above for mortality to maintain

284 the initial population structure. Once dispersal was initiated, individuals were capable of entering a new

285 group as soon as the next day. The dispersing individual moved in a random walk on the two-dimensional

286 lattice of cells representing the different social groups. The lattice was bounded spatially and was not

287 reflective; thus, a dispersing individual that hit a boundary did not move in that time step. When floaters

288 entered a new group, they were capable of transmitting cultural traits as early as the next daily time step

289 of the simulation.

290 In summary, group composition was adjusted to maintain initial, user-specified values by

291 preferentially adding individuals to groups with a deficiency in males or females through births and

292 removing individuals from groups with an excess number of males or females through dispersal events.

\section{Sampling Parameter Space and Simulation Procedures}

295 To explore how different parameters influence cultural dynamics, we undertook multivariate analysis

296 using random sampling. Random sampling was conducted using Latin hypercube sampling (Blower and

297 Dowlatabadi 1994; Seaholm et al. 1988; Rushton et al. 2000), which is a type of stratified Monte Carlo

298 sampling that has been used in epidemiological modeling and is more efficient in this context than

Nunn et al., p. 12 
random sampling regimes or those that include all possible parameter values (Blower and Dowlatabadi

300 1994; Seaholm et al. 1988). Seven parameters were varied in the Latin hypercube sample: transmission

301 model, group size, transmission probability, background mortality, net benefit of the cultural trait, rate of

302 dispersal, and a spatial versus non-spatial dispersal model. Table 1 gives ranges of parameter values. The

303 discretely coded parameters (transmission model, spatial model) were represented as continuously

304 varying traits in the Latin hypercube sample, which were then binned into equal numbers of the discrete

305 traits. We assessed the sample size needed for the Latin hypercube sample by computing the theoretical

306 variance and relative bias of parameter estimates for a range of possible sample sizes. To obtain rough

307 approximations of the aforesaid variance and bias, we fit preliminary models from a few pilot simulation

308 runs. From these computations, we determined that a sample size of 1500 would be sufficient to

309 investigate the effects of parameter variation shown in Table 1.

310 As noted above, each simulation run continued until the prevalence of the cultural trait reached

311 equilibrium or prevalence fell to 0 (i.e., the cultural trait went extinct). For cases in which the trait

312 persisted, equilibrium prevalence was determined empirically. Specifically, the simulation was stopped

313 when six inter-related conditions were met. The cultural trait had (1) spread to all groups (even if

314 subsequently going extinct in one or more groups). The correlation between time and prevalence was (2)

315 non-significant at $\mathrm{P}>0.05$ and (3) explained less than $1 \%$ of the variation over the previous window of

316200 time steps. Similarly, we examined the standard error of overall prevalence and required that it

317 became (4) non-significant at $\mathrm{P}>0.05$ and (5) explained less than $1 \%$ of the variation over the previous

318200 time steps. Finally, we required that (6) the median standard error of overall prevalence was less than

319 the median for 200 time steps. We also examined variation in the time to equilibrium, defined as the first

320 time step in which the estimated equilibrium value was reached, and we investigated factors that led to

321 extinction of the trait. Figure 1 provides an example from one simulation run. The trait spread rapidly and

322 reached an equilibrium prevalence of about 0.80 among individuals in the population after approximately

323500 time steps. From this, the equilibrium prevalence was calculated as 0.798 and the time to equilibrium

324 following infection of all groups occurred on day 504. To satisfy the criteria for identifying equilibrium,

Nunn et al., p. 13 
325 the actual simulation ran for an order of magnitude time longer than the time to equilibrium, with these

326 criteria finally satisfied on day 5537.

\section{Analyses of Simulation Output}

329 We analyzed the output from the simulation using both generalized linear models (GLMs) and regression 330 and classification trees (De'ath and Fabricius 2000; Roff and Roff 2003). We constructed three linear

331 models to explain the simulation outcomes in terms of their parameters. First, all variables were scaled to

332 the unit interval so that the magnitudes of their fitted effects could be compared on an absolute scale. To

333 test for possible interaction effects among the simulation settings, we fit each model using two sets of

334 explanatory variables: a reduced set incorporating only main effects and a full set including all possible

335 interactions. The reduced set was comprised of the seven variables in Table 1 and the particular

336 interaction of background mortality $(m)$ and the cost multiplier of the trait $(c)$, both of which were

337 hypothesized to drive the response. The second set included these factors along with all 27 possible

338 pairwise interactions. For each of the three outcomes, the full and reduced models were compared using

339 the likelihood ratio test and Wald test (McCullagh and Nelder 1989) to assess the significance of the full

340 set of pairwise interactions. All linear models were estimated using standard packages from the R

341 statistical software.

342 Regression and classification trees were calculated for the analysis of extinction probability and

343 time to equilibrium using the Statistics Toolbox in MATLAB v. 7.0. We split impure nodes when the

344 number of observations for that node was 100 for regression trees (time to equilibrium analysis) and 10

345 for classification trees (extinction analysis). After creating an initial tree using the simulation output, we

346 used 10-fold cross-validation to identify the pruning level with the minimal cost (De'ath and Fabricius

347 2000), identified as the tree with the minimum error rate. Using this pruned tree, we calculated the

348 percentage of variance explained by comparing predicted and observed values for the regression trees. 
RESULTS

\section{General Patterns}

353 The simulation model produced a diversity of outcomes, with some traits quickly going extinct and others

354 reaching an equilibrium in which the majority of individuals in the population expressed the trait. These

355 variable outcomes reflected both stochastic effects and the effects of the parameters on the simulation

356 dynamics. Among the 1500 simulations, the cultural trait persisted in $52.5 \%$ of the runs, as defined by the

357 equilibrium conditions described in the Methods; in the remaining simulation runs, the cultural trait went

358 extinct. Of the simulations resulting in trait persistence, the model ran for an average of 1513 time steps

359 (range: 453 to 11,198 time steps). In cases of extinction, the model ran for an average of 842 time steps

360 (range: 1 to 7,477 time steps). In cases of trait persistence, the average proportion of individuals

361 expressing the trait was 0.931 (range: 0.19 to 1.0 ) and the time to reach this equilibrium "prevalence" was

362447 days (range: 18 to 8,893 days). In cases of extinction, the trait spread to an average of 81.2 groups

363 prior to going extinct (range: 1 to 144). Thus, even traits that eventually went extinct often spread widely

364 in the population.

365 The net benefit of the trait varied in the Latin hypercube sample (along with other parameters in

366 Table 1). In general, traits with higher costs tended to more commonly go extinct, while higher benefits

367 favored the establishment of a cultural trait (Figure 2). Remarkably, in $57.7 \%$ of simulations of costly

368 traits, the trait managed to spread to all 144 groups in the population (although not all groups necessarily

369 had the trait simultaneously). In $68 \%$ of these cases of pervasive spread, however, the costly trait

370 subsequently went extinct. Thus, costly social traits spread widely in the simulation, but these traits

371 typically fail to reach a stable equilibrium and eventually go extinct. The analyses below provide more

372 insights into how costs impact trait establishment and spread.

\section{Probability of extinction}

375 We first investigated the factors that influence the probability of extinction. We fit a logistic regression Nunn et al., p. 15 
376 model for the full and reduced variable sets, treating extinction as the binary outcome for all 1,500

377 simulations. Using the Wald test for the significance of the pairwise interaction effects in the full model,

378 we found them to be non-significant $\left(\chi_{27}^{2}=8.31 ; p=0.99\right)$. Consequently, we settled on the reduced

379 model (Table 2). The main drivers in this model are trait cost, background mortality, transmission

380 probability and group size. We found that group size has a strongly negative effect on the probability of

381 extinction, illustrated in the first panel of Figure 3. The effect of trait cost and background mortality on

382 extinction was stronger (based on the parameter estimates) and is shown in the first panel of Figure 4 . The

383 probability of extinction increases with cost, and is further driven by an interaction effect with

384 background mortality. Transmission probability $(\beta)$ had a negative coefficient, indicating that increases in

$385 \beta$ reduced the risk of trait extinction. We also found that higher rates of dispersal reduced the probability

386 of extinction, although this effect only approached significance $(\mathrm{p}=0.07)$. In contrast to these factors, the

387 coefficients associated with the transmission model and spatial models were negligibly small and not

388 significant, indicating that the results were similar across all transmission models and were minimally

389 impacted by either local dispersal or transmission biases.

390 To visualize the effects of the parameters on the probability of extinction, we also ran a

391 classification tree analysis. The resulting tree (Figure 5) revealed that traits were more likely to go extinct

392 at higher costs and higher mortality. The tree also predicts that for beneficial traits, a higher transmission

393 probability $(\beta)$ reduces the probability of extinction. The classification tree analysis confirmed the

394 interaction between costliness of the trait and mortality in the generalized linear model (Table 2), but

395 failed to detect an effect of group size. The tree also provided no evidence for effects of local dispersal or 396 transmission model.

\section{Equilibrium Prevalence}

399 The second set of analyses involved the factors that influenced the proportion of individuals that exhibited 400 the cultural trait at equilibrium (i.e., equilibrium prevalence). For the 787 simulations in which the trait 
401 did not go extinct, we fit a binomial GLM for prevalence, modeling the mean proportion of individuals

402 who have the trait at the end of the simulation. We again tested for interaction effects additional to

403 mortality $x$ cost using the likelihood ratio test and found none to be significant $\left(\chi_{27}^{2}=26.07 ; p=0.49\right)$,

404 leading us to accept the reduced model. The resulting regression estimates are shown in Table 3.

405 As expected, most of the coefficients for the extinction model were reversed in sign for the model

406 describing equilibrium prevalence (i.e., factors that increase prevalence should decrease the probability of

407 extinction). The relative magnitudes of the parameters varied. In the binomial GLM for prevalence, the

408 transmission probability $(\beta)$ had a major impact on prevalence of the cultural trait, with greater values of

$409 \beta$ increasing equilibrium prevalence. Group size (Figure 3), background mortality and trait cost all

410 negatively impacted prevalence, with a strong interaction between mortality and cost (Figure 4). The

411 results were again similar across most transmission and spatial models, although we found a nearly

412 significant effect indicating that consensus transmission results in higher prevalence (as compared to the

413 random model).

414

\section{Time to Equilibrium}

416 We analyzed the factors that influence the speed with which the trait spreads in the population by again

417 focusing on the 787 simulations in which the traits reached an equilibrium. Since the equilibrium times

418 were highly right-skewed, we fit a log-linear model of time to equilibrium. In this case, the full model,

419 with main effects and all pairwise interactions, yielded a significantly better fit than the reduced model,

420 leading the likelihood ratio test to reject the reduced model $\left(\chi_{27}^{2}=49.31 ; p=0.005\right)$. Table 4 shows the

421 most significant effects and interactions from the full model, which explained $75 \%$ of the variation in log-

422 transformed time to equilibrium.

423 The major drivers of time to equilibrium were group size, dispersal rate, cost of the trait and

424 transmission probability. Time to equilibrium decreased with larger group sizes (Figure 3) and greater

425 dispersal rates (Figure 6). A strong negative coefficient indicated that greater transmission probabilities 
426 ( $\beta$ ) also increase the rate at which a cultural trait penetrates a population (Figure 6). Among this set of

427 simulations that resulted in equilibrium, higher costs were associated with more rapid establishment of

428 equilibrium prevalence. The analysis also revealed several significant interaction effects. The

429 combination of greater transmission probability and greater cost and background mortality increased time

430 to equilibrium substantially. While the results were similar across transmission models, time to

431 equilibrium was generally much greater in the spatial model than the non-spatial model, as reflected in

432 both panels of Figure 6.

433 We also ran a regression tree analysis to illustrate the effects of the parameters in Table 1 on the

434 time to equilibrium, which was log-transformed for this analysis (Figure 7). The resulting tree explained

$43564 \%$ of the variation in the time required for a cultural trait to reach equilibrium. Dispersal rate was found

436 at the highest node, as well as in lower parts of the regression tree; in all cases, higher rates of dispersal

437 reduced the time required for a trait to reach equilibrium. Subsequent effects were different at low and

438 high rates of dispersal. When the probability of dispersal was less than 0.0047 , group size played a major

439 role in influencing the time required for a trait to reach equilibrium; with group sizes less than 20.3, the

440 time to equilibrium was predicted to be nearly one order of magnitude higher (based on the log-

441 transformed durations given on the tips of the tree). By contrast, at higher rates of dispersal, a non-spatial

442 model resulted in a marked increase in the rate of trait spread at the population level (predicted values of

4432.50 for local dispersal, versus 2.13 for random dispersal to any group). As expected, a higher

444 transmission probability increased the rate of trait spread. However, prestige and consensus transmission 445 again had no effects on cultural trait dynamics at the population level.

\section{DISCUSSION}

449 In animal societies, most social learning occurs among individuals within groups, and the same was likely 450 to be true of prehistoric human populations. In socially structured populations, establishment of a cultural 
451 trait at the population level requires that the trait spread beyond a single social group, yet with few

452 exceptions (e.g., Henrich and Boyd 1998; Boyd and Richerson 2002), most work on cultural transmission

453 has focused on within-group dynamics. We investigated a set of transmission mechanisms, including

454 biases due to prestige or consensus transmission, and social system parameters to determine which factors

455 influence cultural dynamics in socially structured populations. Among the transmission parameters, the

456 transmission probability $(\beta)$ affected trait persistence and equilibrium levels of trait prevalence, with

457 higher transmission probability resulting in higher prevalence (and also more rapid spread of the trait).

458 Among the social system parameters, increased group size favored the establishment of the trait and

459 enhanced its spread, while increased mortality and trait costs increased the probability of trait extinction

460 and reduced equilibrium prevalence. Remarkably, we found that transmission biases involving prestige or

461 consensus effects had no significant effects on trait dynamics at the population level (although consensus

462 transmission showed evidence for some weak effects on equilibrium prevalence). Another interesting

463 result was that local dispersal slowed the rate of trait spread in the population, but had no significant

464 effects on the probability of extinction or prevalence.

465 One conclusion from these analyses is that the effects of biased transmission involving consensus

466 and prestige effects were minor relative to other factors. Biased transmission may have minor effects

467 because cultural traits can spread rapidly within groups, and the mass-action effect of increasing the

468 number of animals acting as performers may outweigh any minor adjustments in transmission probability

469 caused by prestige or consensus mechanisms. We designed the simulation so that the mean rate of

470 transmission would be approximately equal across the three transmission models that we used. We

471 suggest that effects of biased transmission, if they exist, are weaker than other effects, such as the

472 costliness of the cultural trait.

473 We acknowledge, however, that different implementation of the consensus and prestige models

474 could alter this conclusion. Instead of our simple model of "linear majority rules" for the consensus

475 model, for example, the probability of transmission could have a different shape, including possibly a 
476 more rapid rise at lower prevalence, which could alter the dynamics to speed up trait spread and reduce 477 extinction risk. Similarly, we assumed that the effect of dominance was linear, and that only the rank of 478 the performer was relevant (rather than the difference in the ranks of observer and performer). If we 479 assumed instead that dominants were also more likely to acquire beneficial traits - i.e., that a link exists 480 between dominance rank and the acquisition of beneficial traits - this could impact cultural trait dynamics 481 (e.g., Boyd and Richerson 1985; Boesch and Tomasello 1998; Henrich and Gil-White 2001; Henrich and 482 McElreath 2003). Similarly, we might expect that migration is more likely by lower-ranking individuals. 483 In such a case, the rate of trait spread could slow, as lower-ranking individuals moving into a new group 484 would be less likely to be copied. Thus, our model provides a foundation for exploring the conditions 485 under which prestige and consensus mechanisms have an impact on par with the effect of social system 486 parameters.

An almost limitless set of cultural transmission mechanisms is possible (e.g., Laland 2004), and 488 thus we were forced to select a small subset of key factors that might bias transmission (Boesch and 489 Tomasello 1998; Henrich and McElreath 2003). We further aimed to implement these transmission 490 models as simply as possible, for example by using linear transformations of the probability of 491 transmission based on dominance rank of culturally-skilled individuals (prestige model) or the percentage 492 of animals in the group that expressed the trait (consensus model). Future research could consider variants 493 on these models, and also constraints. For example, there could be greater opportunities for transmitting 494 traits within the sexes than between them (e.g., clothing fashions). Similarly, social groups themselves are 495 often composed of networks of interactions involving kin, alliances and sexual partners, and some traits 496 might be transmitted vertically from mother to offspring. Age effects might also be important, with 497 transmission to an observer more likely during age-specific periods when learning is more likely, or the 498 behaviors themselves only expressed at a particular life stage; such effects would be expected to slow the 499 spread of the cultural trait. It would also be interesting to investigate competition among traits that have 500 different transmission mechanisms or benefits to individuals with the traits. Finally, it is worth keeping in 
501 mind that the prestige and consensus models are not mutually exclusive. Although we treated them 502 separately here, it might be interesting to investigate their combined effects on cultural trait dynamics.

503 The social factors that we investigated have clear analogies to the spread of infectious disease in

504 socially structured populations, particularly for costly cultural traits that can negatively impact fitness.

505 Returning to the case of individuals copying dominants, for example, similar patterns can be found with

506 sexually transmitted diseases (STDs). In epidemiological models of STDs in animals, more dominant

507 individuals are more likely to be infected, and thus more likely to spread the disease (Thrall et al. 2000;

508 Kokko et al. 2002). Similarly, disease spread can be impacted by group size, patterns of dispersal, and

509 mortality rates (Anderson and May 1991; Wilson et al. 2003; Nunn and Altizer 2006).

510 However, important differences exist between the spread of cultural traits and infectious disease,

511 particularly with regard to the selective benefits of many cultural traits (in comparison to costs usually

512 associated with disease). As compared to disease transmission, for example, cultural evolution in socially

513 structured populations is likely to set up a group selection scenario, in which advantageous cultural traits

514 could lead to larger groups and higher rates of dispersal (Wilson 1983; Soltis et al. 1995). In addition,

515 cultural traits in animals and early humans tend to spread directly between individuals in close proximity,

516 while infectious diseases can be transmitted indirectly (e.g., through vectors or contaminated soil). Lastly,

517 innovation is possible in cultural systems, even if it is often "primed" by previous innovations or cultural

518 structures, whereas infectious diseases do not typically arise de novo in a population (although they could

519 appear to do so when spillover from a reservoir host occurs, or when hybridization among pathogens

520 opens up new hosts to exploit). In other words, you do not actually have to have direct contact with an

521 “infected" individual to get a good idea; individual learning can also play a role, and is ultimately

522 responsible for the origin of cultural behaviors.

523 A beneficial cultural trait is expected to spread rapidly and reach high prevalence, and our

524 simulations confirmed this expectation under a wide range of conditions. Advantageous behaviors are

525 also likely to reduce the likelihood of group extinction, which could create opportunities for group

526 selection in natural situations. On the other hand, one can easily think of cultural traits that are clearly not Nunn et al., p. 21 
527 advantageous for survival, yet spread throughout populations. These are superficially similar to

528 establishment of infectious diseases, which entail a cost to the host but still can reach a stable equilibrium.

529 Our simulations suggest that costly cultural traits can spread widely, but as costs increase the probability

530 of extinction also increases.

531 The results of our analysis suggest that the explosion of cultural behaviors and variants in human

532 evolution should have resulted when group size, contact between groups and the benefits of cultural traits

533 increased. Many cultural traits in humans are technological. Hence, these traits would be likely to carry a

534 very strong benefit, favoring their establishment in both species. Second, the higher technological skills

535 seen in human evolution, with the inclusion of many stone tools, could reasonably have led to a reduction

536 in mortality rates. This would have favored the further development of larger social groups, which as we

537 saw in our analysis, favor the establishment of cultural traits. Lastly, in comparison to other apes, humans

538 live in more dispersed social groups, in much larger home ranges, and with regular contact including

539 more than only direct neighbors; these social groups likely had more contact with other groups as trade

540 took place. Our results suggest that these contacts would have increased the rate at which cultural traits

541 spread, and might have reduced the probability that they went extinct.

542 To conclude, it is useful to return to the four questions that we posed in the Introduction. The

543 simulations revealed that local dispersal increases the time required for a trait to reach equilibrium

544 (Question 1), and that cultural traits are buffered from extinction in larger groups (Question 2). We also

545 found that higher rates of dispersal increase the rate of trait spread in the population, with weaker effects

546 (approaching significance) on the probability of extinction. In terms of mortality, we found that mortality

547 rates have an impact on cultural dynamics, including through effects of the cultural trait on mortality itself

548 (Question 3). Thus, higher costs of the trait and higher background mortality increase extinction

549 probability and reduce the prevalence of the trait. Lastly, we found that the rate of transmission impacts

550 all of the outcome variables that we examined, but that transmission mechanisms involving prestige or

551 consensus had no statistically discernible effects on trait dynamics (Question 4). As noted above, this

552 conclusion could be sensitive to how prestige and consensus transmission were implemented, and

Nunn et al., p. 22 
553 therefore should be explored further in future research. Along similar lines, it would be interesting to

554 explore other transmission mechanisms that might influence the spread of traits among contact networks

555 within groups, including vertical transmission, sex- and age-specific transmission, and patterns of kinship.

556

Nunn et al., p. 23 


\section{Acknowledgements}

558 We thank Richard McElreath, Mathias Franz, Derek Roff, Monique Borgerhoff Mulder and members of

559 the "culture group" at UC Davis and the "primate group" at Harvard for helpful suggestions and

560 discussion. Markus Bayer provided help compiling the MATLAB code and using the computer cluster.

561 This research was supported by the Max Planck Society.

562

563 


\section{References}

Anderson, R. M. \& May, R. M. 1979. Population biology of infectious diseases: Part 1. Nature, 280, 361-367.

Anderson, R. M. \& May, R. M. 1981. The population-dynamics of micro-parasites and their invertebrate hosts. Philosophical Transactions of the Royal Society of London Series B-Biological Sciences, 291, 451-524.

Anderson, R. M. \& May, R. M. 1991. Infectious diseases of humans: Dynamics and control: Oxford

Blower, S. M. \& Dowlatabadi, H. 1994. Sensitivity and uncertainty analysis of complex-models of disease transmission - an HIV model, as an example. International Statistical Review, 62, 229-243.

Boesch, C., Marchesi, P., Marchesi, N., Fruth, B. \& Joulian, F. 1994. Is nut cracking in wild chimpanzees a cultural behaviour? Journal of Human Evolution, 26, 325-338.

Boesch, C. \& Tomasello, M. 1998. Chimpanzee and human cultures. Current Anthropology, 39, 591614.

Boesch, C. \& Boesch-Achermann, H. 2000. The chimpanzees of the Tai forest. Oxford: Oxford University Press.

Boesch, C. 2003. Is culture a golden barrier between human and chimpanzee? Evolutionary Anthropology, 12, 82-91.

Boots, M. \& Sasaki, A. 1999. 'Small worlds' and the evolution of virulence: Infection occurs locally and at a distance. Proceedings of the Royal Society of London - Series B: Biological Sciences, 266, 19331938.

Boyd, R. \& Richerson, P. J. 1985. Culture and the evolutionary process. Chicago: Chicago University Press.

Boyd, R. \& Richerson, P. J. 2002. Group beneficial norms can spread rapidly in a structured population. Journal of Theoretical Biology, 215, 287-296.

Nunn et al., p. 25 
Carlsson-Graner, U.\& Thrall, P. H. 2002. The spatial distribution of plant populations, disease dynamics and evolution of resistance. OIKOS, 97, 97-110.

591 De'ath, G. \& Fabricius, K. E. 2000. Classification and regression trees: A powerful yet simple technique 592 for ecological data analysis. Ecology, 81, 3178-3192.

593 Franz, M. \& Nunn, C. L. 2009. Network-based diffusion analysis: A new method for detecting social 594 learning. Proceedings of the Royal Society B, in press.

595 Gandon, S., Capowiez, Y., Dubois, Y., Michalakis, Y. \& Olivieri, I. 1996. Local adaptation and gene596 for-gene coevolution in a metapopulation model. Proceedings of the Royal Society of London Series B-Biological Sciences, 263, 1003-1009.

Grimm, V.\& Railsback, S. F. 2005. Individual-based modeling and ecology. Princeton: Princeton $601 \quad$ University Press.

602 Henrich, J. \& Boyd, R. 1998. The evolution of conformist transmission and the emergence of betweengroup differences. Evolution and Human Behavior, 19, 215-241.

604 Henrich, J. \& Gil-White, F. J. 2001. The evolution of prestige - freely conferred deference as a 605 mechanism for enhancing the benefits of cultural transmission. Evolution and Human Behavior, 22, $606 \quad 165-196$.

607 Henrich, J. \& McElreath, R. 2003. The evolution of cultural evolution. Evolutionary Anthropology, 12, $608 \quad 123-135$.

609 Hunt, G. R. 2003. Diversification and cumulative evolution in New Caledonian crow tool manufacture. $610 \quad$ Proceedings of the Royal Society B: Biological Sciences, 270, 867-874.

611 Kawai, M. 1965. Newly acquired pre-cultural behavior of the natural troop of Japanese monkeys on $612 \quad$ Koshima Islet. Primates, 6, 1-30.

613 Kawamura, S. 1959. The process of sub-culture propagation among Japanese monkeys. Primates, 2, 43614 60. 
Kokko, H., Ranta, E., Ruxton, G. \& Lundberg, P. 2002. Sexually transmitted disease and the evolution of mating systems. Evolution, 56, 1091-1100.

617 Laland, K. N. 2004. Social learning strategies. Learning \& Behavior, 32, 4-14.

618 Leca, J. B., Gunst, N. \& Huffman, M. A. 2007. Japanese macaque cultures: Inter- and intra-troop 619 behavioural variability of stone handling patterns across 10 troops. Behaviour, 144, 251-281.

620 May, R. M. \& Anderson, R. M. 1979. Population biology of infectious diseases: Part II. Nature, 280, $621 \quad 455-461$.

622 McCullagh, P. \& Nelder, J. 1989. Generalized linear models: Chapman \& Hall.

623 Mesoudi, A., Whiten, A. \& Laland, K. N. 2004. Perspective: Is human cultural evolution darwinian?

624 Evidence reviewed from the perspective of the Origin of Species. Evolution, 58, 1-11.

625 Nunn, C. L. \& Altizer, S. M. 2006. Infectious diseases in primates: Behavior, ecology and evolution. 626 Oxford: Oxford University Press.

627 Nunn, C. L., Thrall, P. H., Harcourt, A. H. \& Stewart, K. 2008. Emerging infectious diseases and 628 animal social systems. Evolutionary Ecology, 22, 519-543.

629 O'Keefe, K. J. \& Antonovics, J. 2002. Playing by different rules: The evolution of virulence in $630 \quad$ sterilizing pathogens. . American Naturalist, 159, 597-605.

631 Roff, D. A. \& Roff, R. J. 2003. Of rats and Maoris: A novel method for the analysis of patterns of 632 extinction in the New Zealand avifauna before European contact. Evolutionary Ecology Research, 5, $633 \quad 759-779$.

634 Roy, B. A. \& Kirchner, J. W. 2000. Evolutionary dynamics of pathogen resistance and tolerance. $635 \quad$ Evolution, 54, 51-63.

636 Rushton, S. P., Lurz, P. W. W., Gurnell, J. \& Fuller, R. 2000. Modelling the spatial dynamics of 637 parapoxvirus disease in red and grey squirrels: A possible cause of the decline in the red squirrel in 638 the UK? Journal of Applied Ecology, 37, 997-1012.

639 Seaholm, S. K., Ackerman, E. \& Wu, S. C. 1988. Latin hypercube sampling and the sensitivity analysis 640 of a monte-carlo epidemic model. International Journal of Bio-Medical Computing, 23, 97-112. 
641 Soltis, J., Boyd, R. \& Richerson, P. J. 1995. Can group-functional behaviors evolve by cultural group

642 selection? An empirical test. Current Anthropology, 36, 473-494.

643 Thrall, P. H. \& Antonovics, J. 1995. Theoretical and empirical-studies of metapopulations - population

644 and genetic dynamics of the Silene-Ustilago system. Canadian Journal of Botany-Revue Canadienne

645 De Botanique, 73, S1249-S1258.

646 Thrall, P. H., Antonovics, J. \& Dobson, A. P. 2000. Sexually transmitted diseases in polygynous mating

647 systems: Prevalence and impact on reproductive success. Proceeding of the Royal Society London B, $648 \quad 267,1555-1563$.

649 van der Post, D. J. \& Hogeweg, P. 2006. Resource distributions and diet development by trial-and-error $650 \quad$ learning. Behavioral Ecology and Sociobiology, 61, 65-80.

651 Van der Post, D. J. \& Hogeweg, P. 2008. Diet traditions and cumulative cultural processes as side652 effects of grouping. Animal Behaviour, 75, 133-144.

653 van Schaik, C. P., Deaner, R. O.\& Merrill, M. Y. 1999. The conditions for tool use in primates:

654 Implications for the evolution of material culture. Journal of Human Evolution, 36, 719-741.

655 van Schaik, C. P.\& Pradhan, G. R. 2003. A model for tool-use traditions in primates: Implications for 656 the coevolution of culture and cognition. Journal of Human Evolution, 44, 645-664.

657 Wilson, D. S. 1983. The group selection controversy: History and current status. Annual Review of $658 \quad$ Ecology and Systematics, 14, 159-187.

659 Wilson, K., Knell, R., Boots, M. \& Koch-Osborne, J. 2003. Group living and investment in immune 660 defence: An interspecific analysis. Journal of Animal Ecology, 72, 133-143. 
663 Figure 1. Detecting equilibrium. Plot shows output from one simulation run using default parameters.

664 The text provides details on equilibrium prevalence and time step at which this was first reached, as

665 calculated by the simulation program.

Figure 2. Trait persistence in relation to net benefits of the cultural trait. Bars indicate number of

668 cases in which the trait reached an equilibrium, as compared to the alternative of going extinct. Increasing

669 benefits are shown to the left of the central line, while increasing costs are shown to the right. Results are

670 based on the output from 1500 simulations. As the Latin hypercube sample provided a flat distribution for

671 the values, including costs of the trait shown along the x-axis, this plot reveals that higher costs are

672 associated with higher extinction, but that some costly traits nonetheless reach an equilibrium.

674 Figure 3. Modeled extinction, prevalence and time to equilibrium by size. The three panels show the

675 modeled extinction rate, prevalence and time to equilibrium by scaled group size. The rates correspond to

676 default values of the other settings.

Figure 4. Modeled extinction and prevalence by background mortality and trait cost. The two panels

679 show the modeled extinction and prevalence rates across a range of cost levels at two different

680 background mortality rates (scaled on the interval 0 to 1 ). The other settings are at default values.

682 Figure 5. Classification tree for extinction. Extinction is indicated as a dichotomous trait on the tips of 683 the tree, where "survive" indicates that the trait is predicted to reach an equilibrium rather than go extinct.

685 Figure 6. Modeled time to equilibrium by dispersal rate. The plots show the effects of dispersal rate, 686 transmission probability and spatial model on time to equilibrium. The other settings are at default values. 
688 Figure 7. Regression tree for time to equilibrium. Time to equilibrium is log-transformed, with 689 predicted values shown at the tips of the tree. 
Table 1. Parameter values investigated in the simulation.

\begin{tabular}{|l|l|c|}
\hline Symbol & \multicolumn{1}{|c|}{ Definition } & Range of Values \\
\hline$g$ & average number of females in groups, $g_{m}$ & 4 to 40 \\
\hline$d$ & baseline probability of dispersal per day & 0.0001 to 0.02 \\
\hline$\beta$ & per-contact transmission probability & 0.0001 to 0.04 \\
\hline$m$ & baseline mortality rate per day & 0.0001 to 0.04 \\
\hline$c$ & benefit or cost of cultural trait (multiplier for $m$ ) & 0.001 to 2 \\
\hline$S$ & spatial vs. non-spatial model (categorical) & 0,1 \\
\hline$T$ & transmission model (categorical, corresponding to & $0,1,2$ \\
\hline
\end{tabular}

691 
692 Table 2. Parameter estimates in the logistic regression model for extinction probability.

\begin{tabular}{|l|r|r|r|}
\hline \multicolumn{1}{|c|}{ Parameter } & Estimate & Std. Error & p-value \\
\hline Intercept & -3.35 & 0.68 & $<0.001$ \\
\hline$g$ & -1.42 & 0.38 & $<0.001$ \\
\hline$d$ & -0.67 & 0.37 & 0.070 \\
\hline$\beta$ & -1.81 & 0.39 & $<0.001$ \\
\hline$m$ & 0.04 & 1.03 & 0.971 \\
\hline$c$ & 6.01 & 0.92 & $<0.001$ \\
\hline$T$ (consensus vs. random) & 0.37 & 0.26 & 0.153 \\
\hline$T$ (prestige vs. random) & 0.36 & 0.27 & 0.178 \\
\hline$S$ (spatial vs. non-spatial) & 0.16 & 0.21 & 0.453 \\
\hline$m *$ c & 7.93 & 1.92 & $<0.001$ \\
\hline
\end{tabular}

693

694 
694 Table 3. Parameter estimates in the binomial GLM model for trait prevalence.

\begin{tabular}{|l|r|r|r|}
\hline Parameter & Estimate & Std. Error & p-value \\
\hline Intercept & 2.43 & 0.18 & $<0.001$ \\
\hline$g$ & 1.84 & 0.12 & $<0.001$ \\
\hline$d$ & 0.12 & 0.11 & 0.293 \\
\hline$\beta$ & 2.91 & 0.13 & $<0.001$ \\
\hline$m$ & -0.79 & 0.24 & 0.001 \\
\hline$c$ & -1.95 & 0.31 & $<0.001$ \\
\hline$T$ (consensus vs. random) & 0.14 & 0.08 & 0.065 \\
\hline$T$ (prestige vs. random) & -0.09 & 0.08 & 0.214 \\
\hline$S$ (spatial vs. non-spatial) & 0.04 & 0.06 & 0.455 \\
\hline$m *$ c & -4.89 & 0.58 & $<0.001$ \\
\hline
\end{tabular}

695

696 
696 Table 4. Significant parameter estimates in the log-linear model for time to equilibrium.

\begin{tabular}{|l|r|r|r|}
\hline Parameter & Estimate & Std. Error & p-value \\
\hline Intercept & 7.52 & 0.22 & $<0.001$ \\
\hline$g$ & -1.68 & 0.26 & $<0.001$ \\
\hline$d$ & -1.21 & 0.25 & $<0.001$ \\
\hline$\beta$ & -1.56 & 0.27 & $<0.001$ \\
\hline$c$ & -1.23 & 0.33 & $<0.001$ \\
\hline$S($ spatial vs. non-spatial $)$ & 0.82 & 0.15 & $<0.001$ \\
\hline$d * \beta$ & -0.47 & 0.23 & 0.042 \\
\hline$\beta * m$ & 0.69 & 0.23 & 0.003 \\
\hline$\beta * c$ & 1.38 & 0.33 & $<0.001$ \\
\hline$(27$ others $)$ & & & \\
\hline
\end{tabular}

697 
Fig. 1

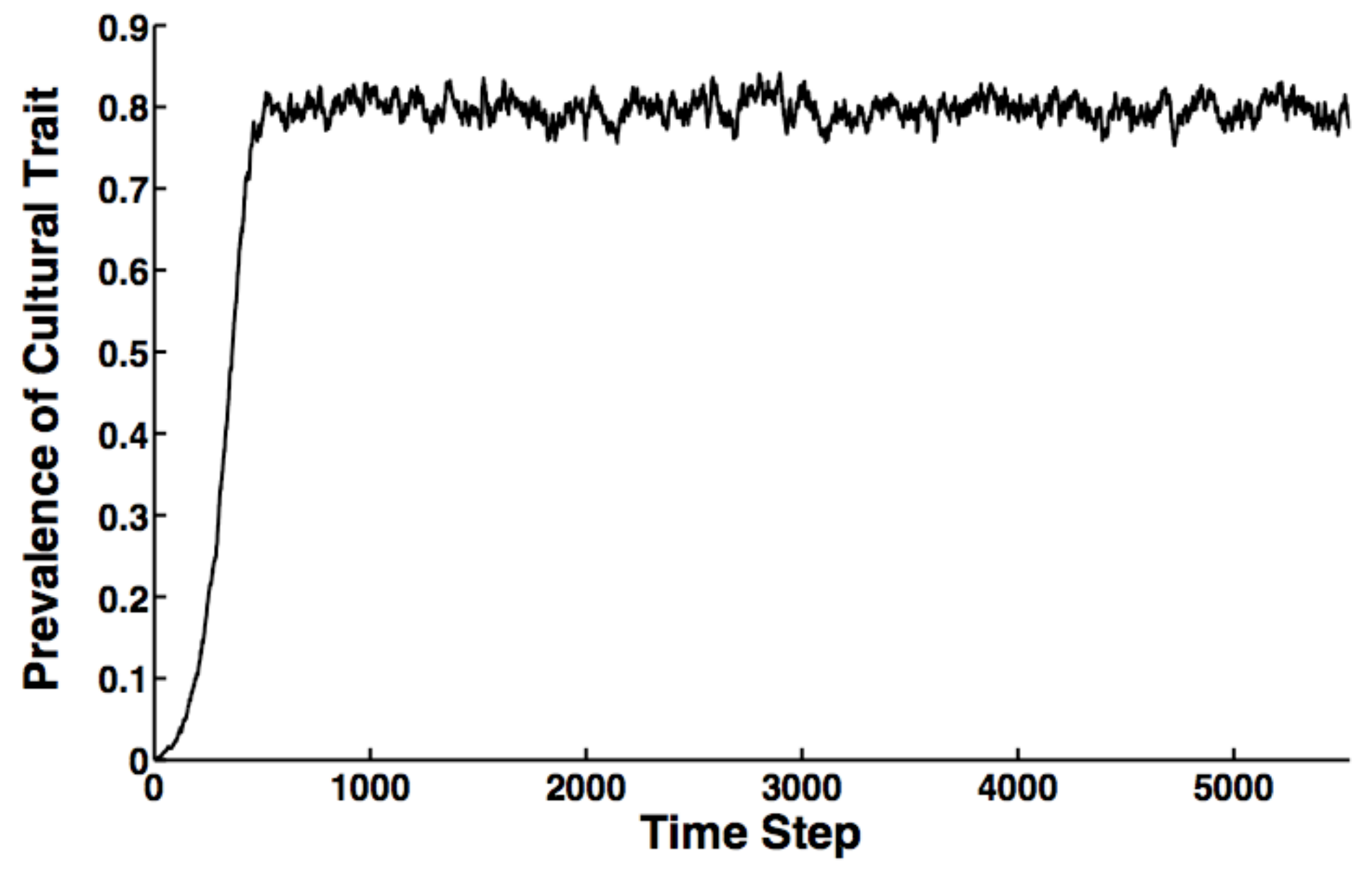


Fig. 2

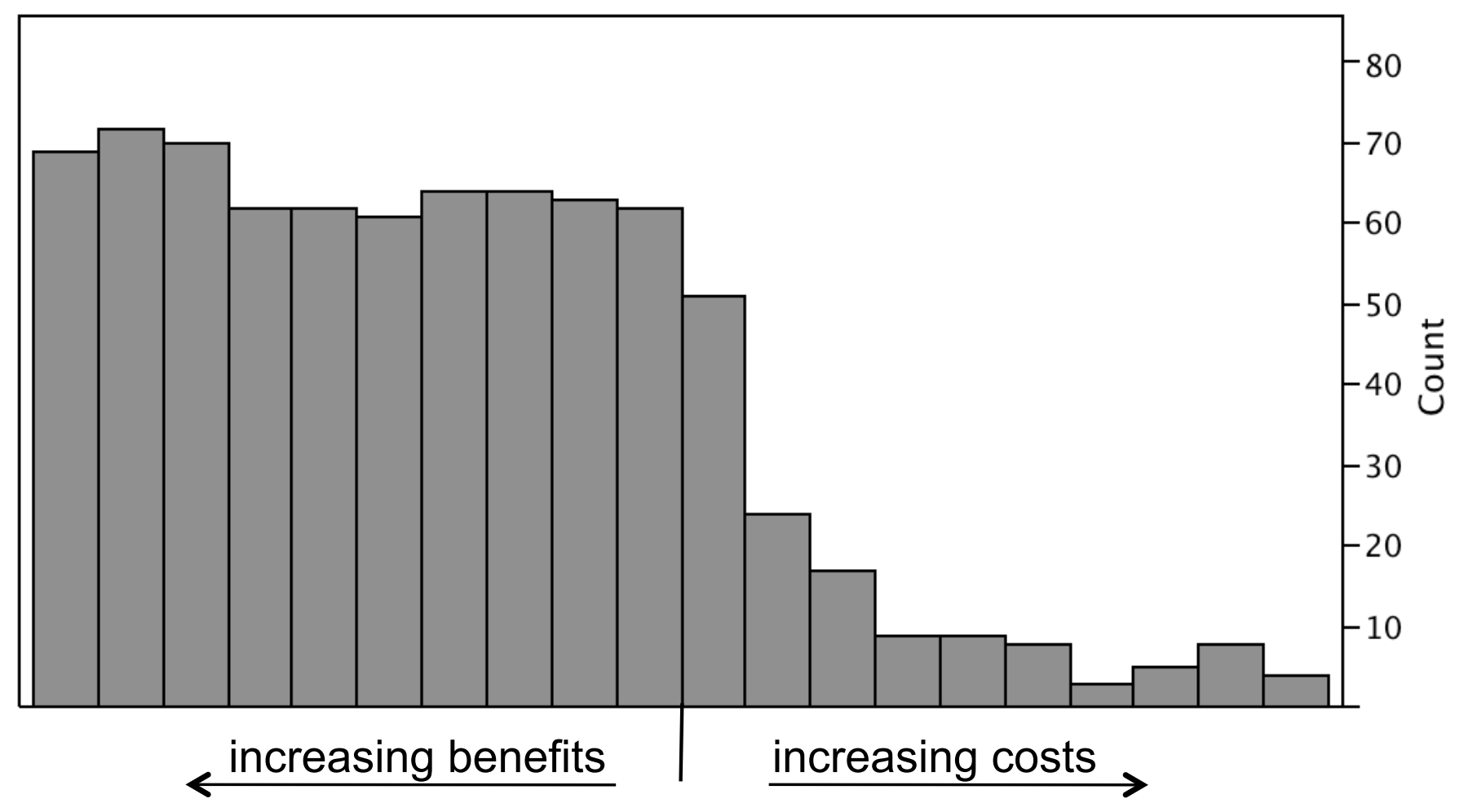


Fig. 3
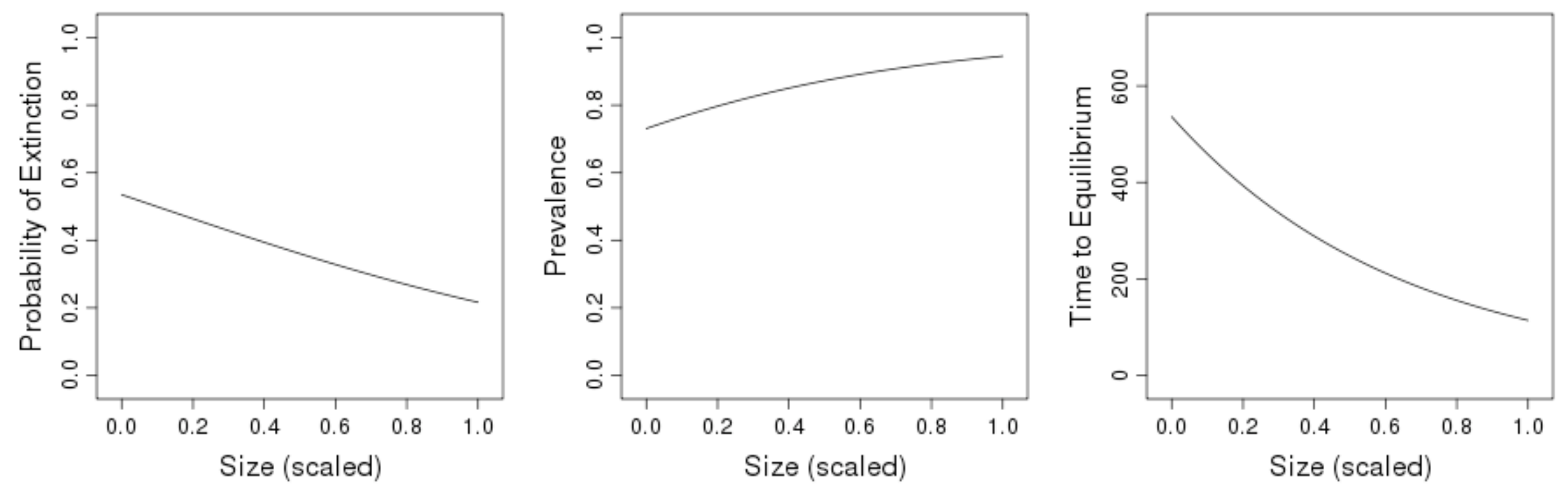
Fig. 4

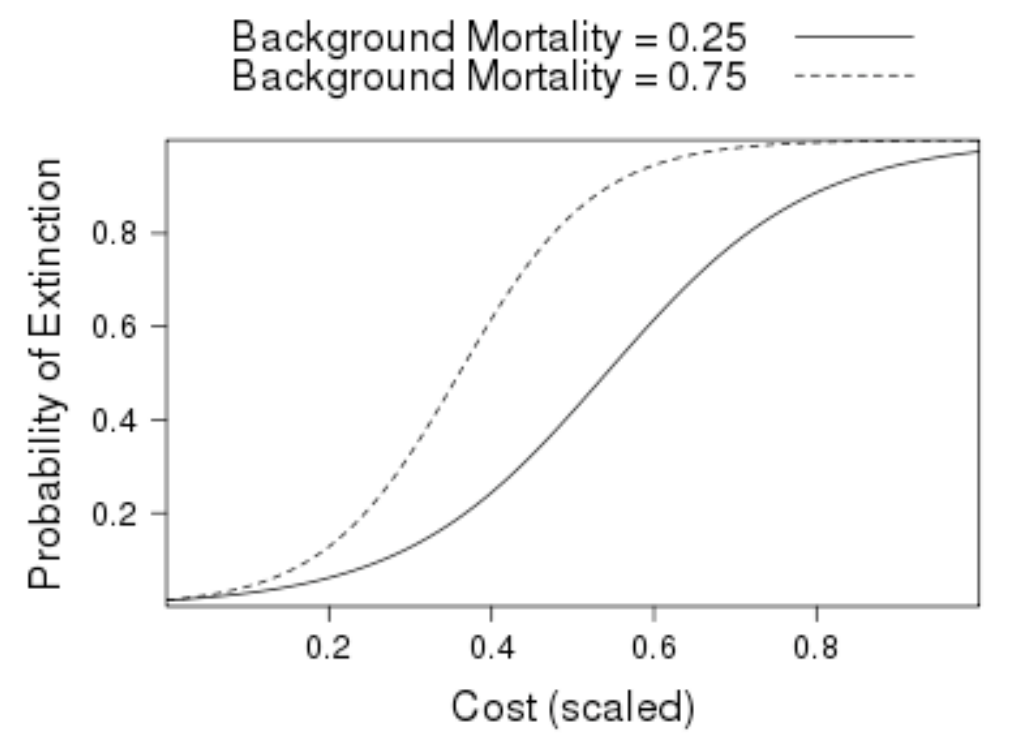

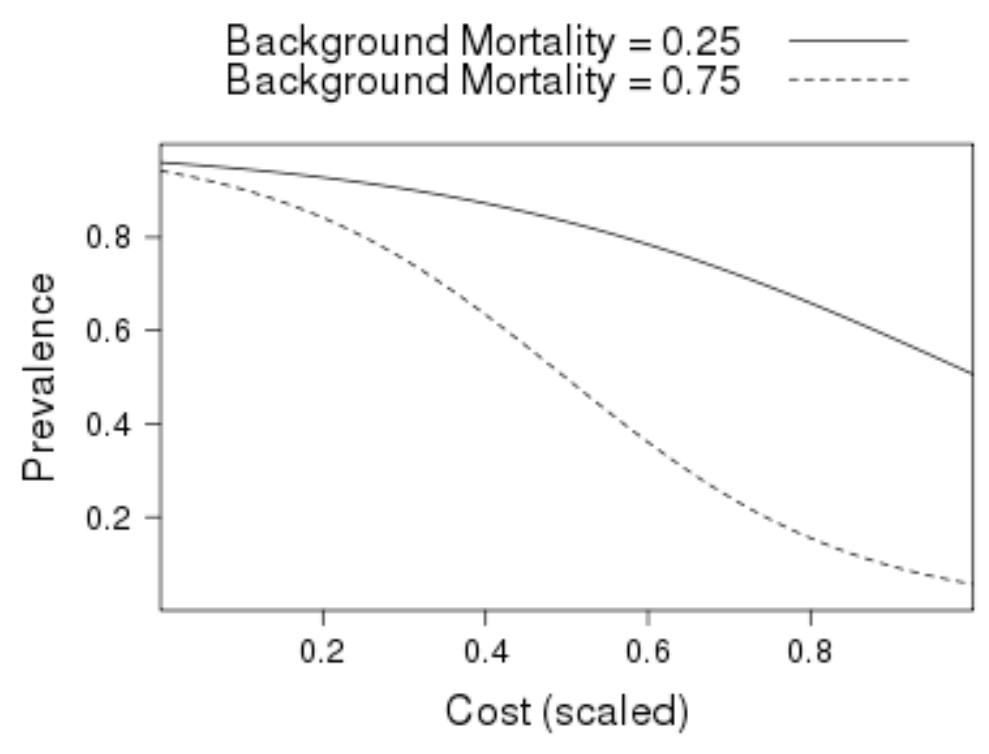


Fig. 5

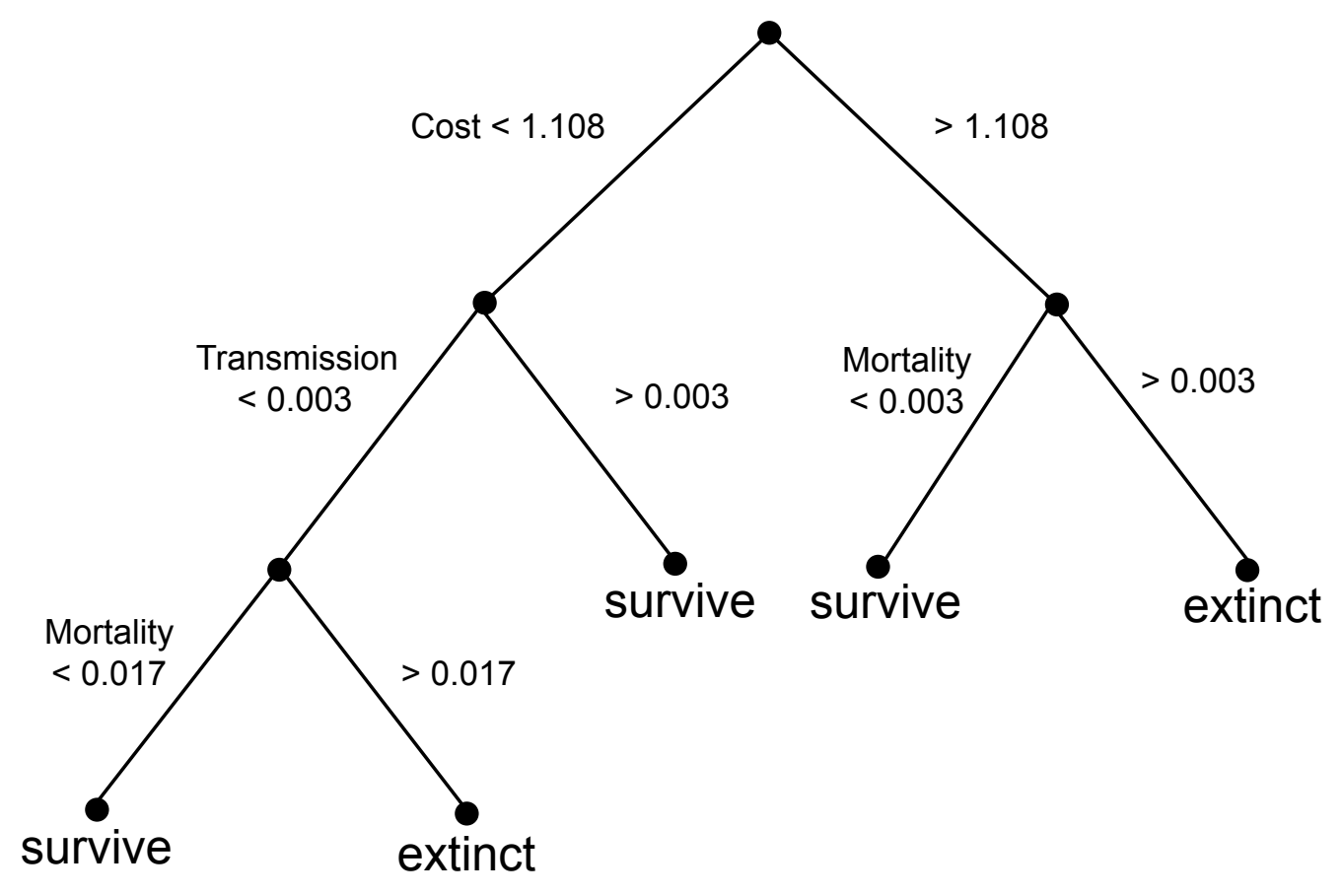


Fig. 6

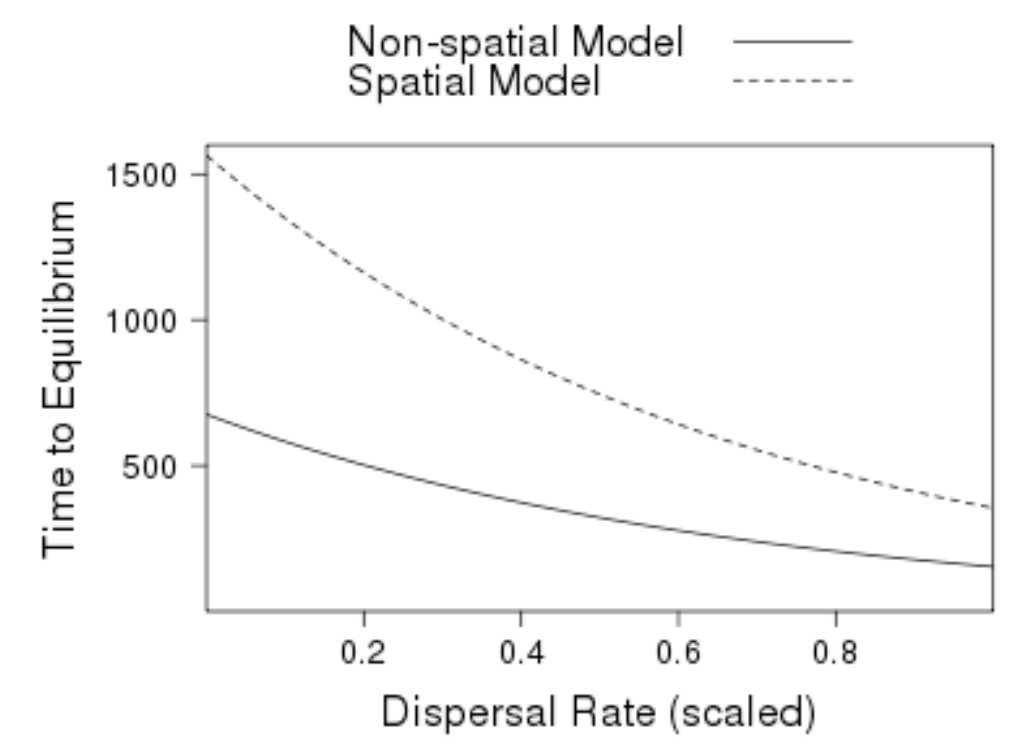

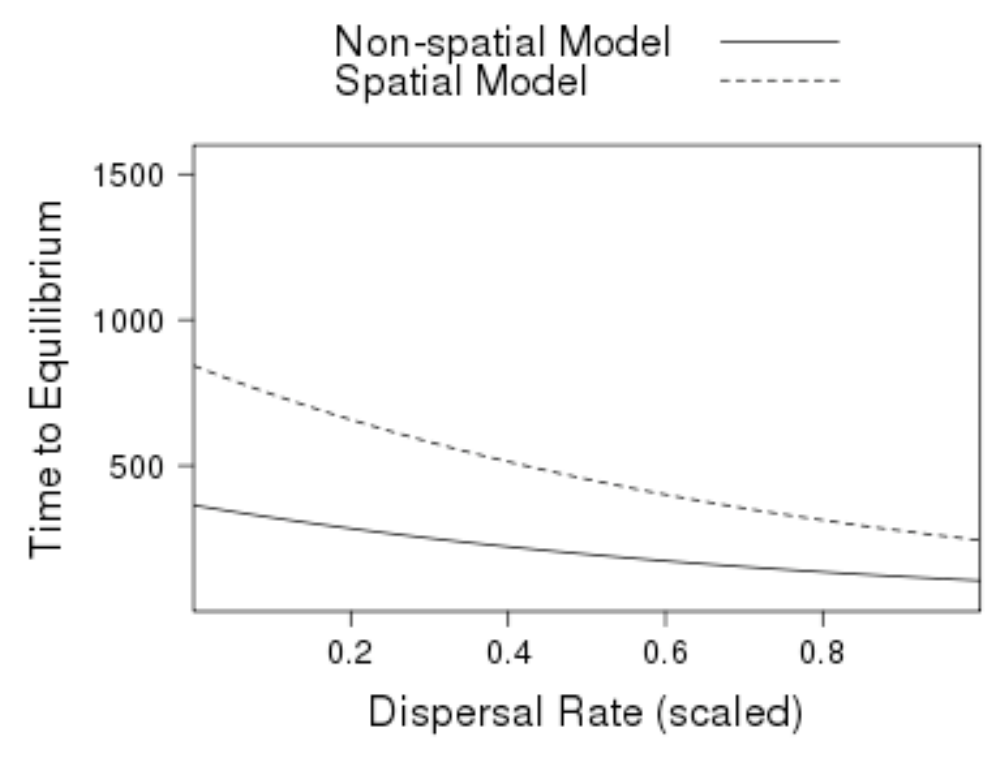

Non-spatial Model

Spatial Model

Dispersal Rate (scaled) 
Fig. 7

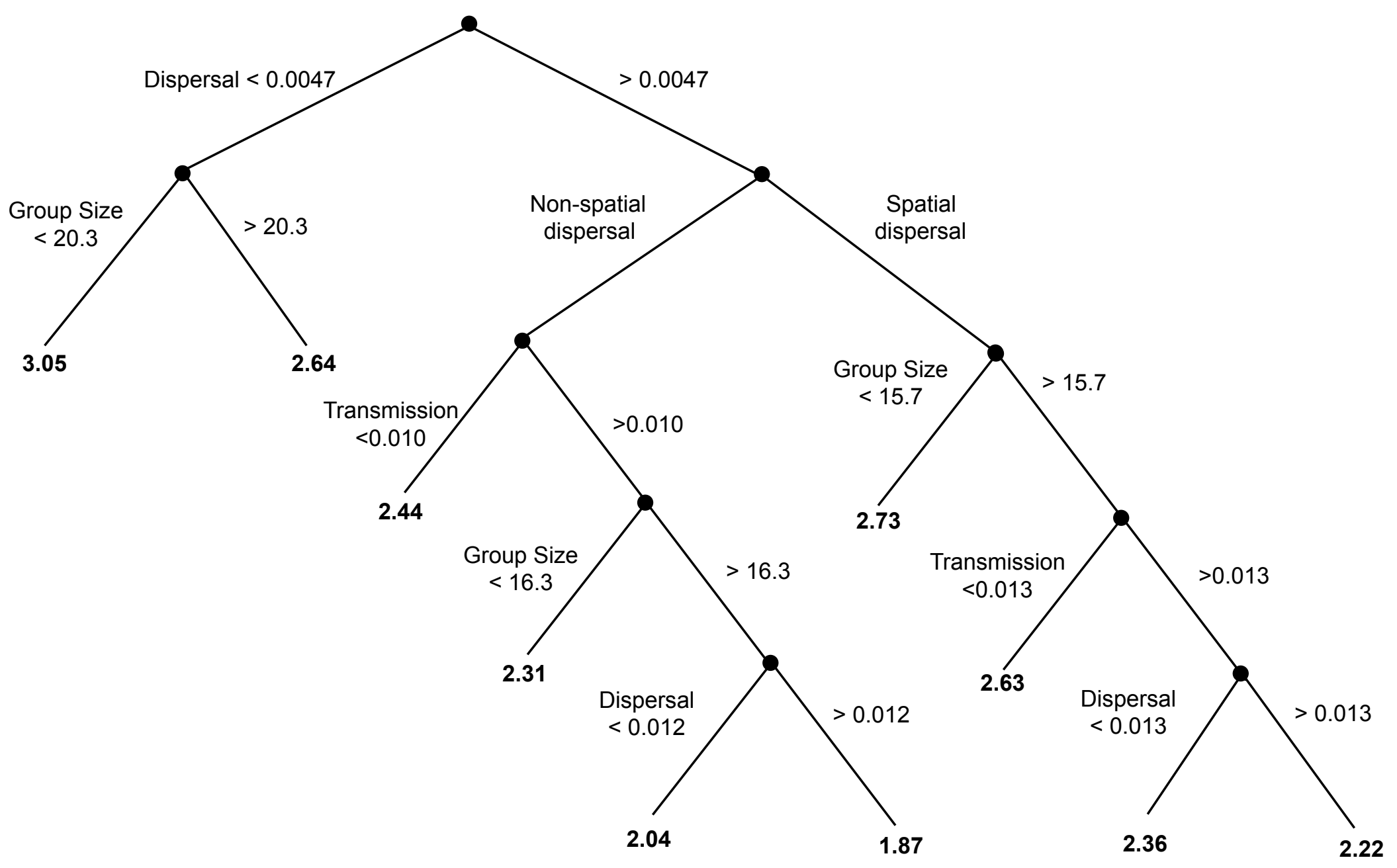

